\title{
Importance of Genotoxicity \& S2A guidelines for genotoxicity testing for pharmaceuticals:
}

\author{
Shaily Umang Shah
}

Genotoxicity is a word in genetics defined as a destructive effect on a cell's genetic material (DNA, RNA) affecting its integrity. Genotoxins are mutagens; they can cause mutations. Genotoxins include both radiation and chemical genotoxins. A substance that has the property of genotoxicity is known as a genotoxin. There are three primary effects that genotoxins can have on organisms by affecting their genetic information. Genotoxins can be carcinogens, or cancer-causing agents, mutagens, or mutation-causing agents, or teratogens, birth defect-causing agents. In most cases, genotoxicity leads to mutations in various cells and other bodily systems. Mutations can lead to a host of other problems, from cancer to a wide variety of different diseases. Mutations can come in many different forms; genetic information can be duplicated, deleted, or inserted.

Genotoxic

Risk

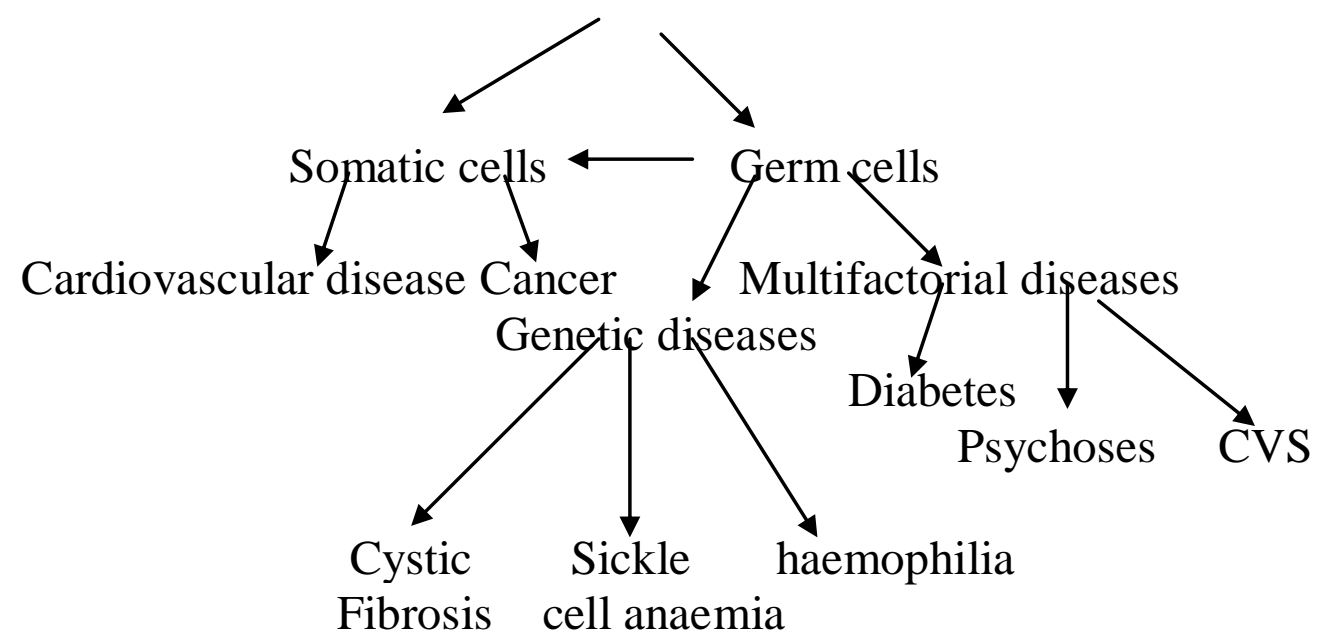

\section{Importance Of Genotoxicity Testing:}

Regulatory authorities all over the world require data on the genotoxic potential of new drugs, as part of the safety evaluation process. The pre-clinical studies are generally conducted to obtain the basic toxicological profile of new chemical entities (NCE). The toxicological data are used to evaluate the safety and efficacy of NCE, which will help in predicting the drug's likely risk/benefit assessment in New Drug Application (NDA) process. Genotoxicity assays have become an integral component of regulatory requirement. In addition to it, many people in India are not aware of genotoxicity that it has now become mandatory to include it in drug master file required by European and United States regulatory authorities.

Genotoxicity testing of new chemical entities (NCE) is generally used for hazard identification with respect to DNA damage and its fixation. These damages can be manifested in the form of gene mutation, structural chromosomal aberration, recombination and numerical changes. These changes are responsible for heritable effects documented that somatic mutations can also play an important role in malignancy

These tests have been used mainly for the prediction of carcinogenicity and genotoxicity because compounds, which are positive in these tests, have the potential to be human carcinogens and/or mutagens.

\section{Classification Of Carcinogens:}

The existing systems of classification of carcinogens should include a distinction between genotoxic and non-genotoxic chemicals. For non-genotoxic chemicals, permissible exposure levels can be derived at which no relevant human cancer risks are anticipated. While genotoxic carcinogens can induce chromosomal effects without mutagenic action, non-DNA-reactive genotoxins affecting topoisomerase or the spindle, or those having an exclusively aneugenic effect can be carcinogenic only at high, toxic doses. Specific mechanisms of 
clastogenicity and processes of carcinogenesis based on reactive oxygen have practical thresholds. Since reactive oxygen species (ROS) are generally genotoxic, the question is whether chemicals that increase ROS production will add to endogenously produced background levels and lead to nonlinear dose-effect relationships. Taking into account the presence of endogenous carcinogens, it is now becoming evident that carcinogenic risk extrapolation to low doses must be considered according to the mode of action.

Genotoxicity, especially when of local nature may only be relevant under conditions of sustained local tissue damage and the associated increased cell proliferation. Cases in point are formaldehyde and vinyl acetate. Defining practical thresholds and health-based exposure limits for these two compounds may prove justified. High doses of reactive oxygen species (ROS) or ROS promoters are clearly toxic. ROS are involved in many forms of tissue damage such as ischemia-reperfusion, atherosclerosis, radiation injury, aging and carcinogenesis. Generally, "oxidative stress" is an important mechanism of indirect genotoxicity that is triggered by exposure to exogenous factors such as UV, ionising radiation, anoxia and hyperoxia. Other pathways are mediated by chemicals producing reactive oxygen species .Paraquat and certain oxidants (potassium bromate, hydrogen peroxide) are the classical examples in this respect. Other exogenous sources of ROS are tobacco smoke, fatty acids, transition metals, ethanol, and redox cycling compounds or physical irradiation by multiple sources. ROS interact with critical molecules within cells and with intracellular signalling, leading to cell death, mutagenesis and toxicities associated with lipid peroxidation. Increased oxidative stress and excessive ROS production cause damage to DNA modifying the base and altering DNA strands, and can contribute to cancer.

\section{Agents capable of causing direct or indirect damage to DNA}

- Electrophilic species forming covalent adducts to DNA

E.g. alkylatingagents

Arylnitrenium ions

Diol epoxides of PAH

Etc.

- UV and ionising radiations

- Reactive oxygen species

- Topoisomerase inhibitors

- Nucleoside analogues

- Protein synthesis inhibitors

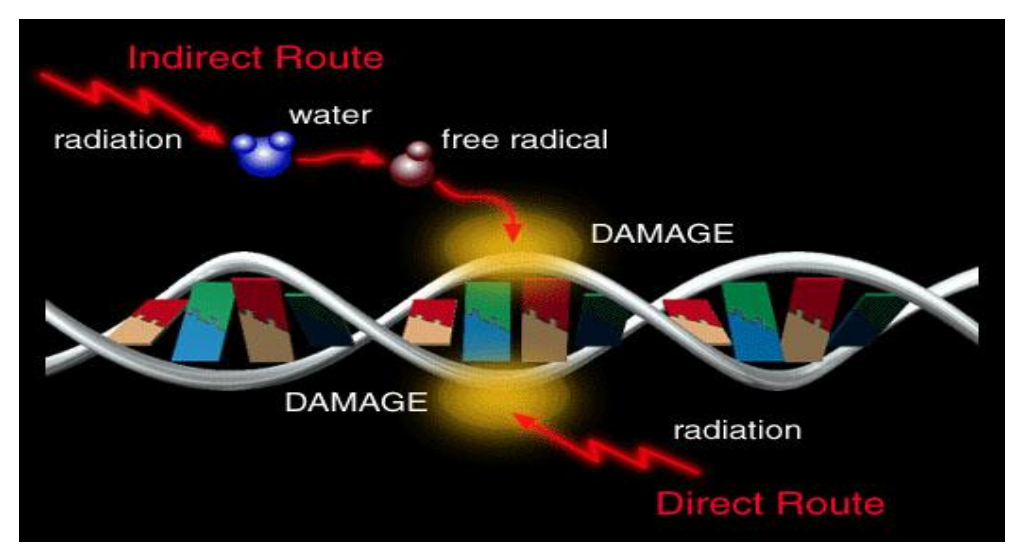

In the EU directives, carcinogens are classified according to a system which was introduced in the early eighties and which was based on the existing national systems. The decision making completely separates the sequential processes of "hazard assessment", which is directed towards classification, from labelling and "risk assessment" which is directed towards standard setting. Both the categorisation and the procedures for low-dose extrapolation of the risk of chemical carcinogens are now being discussed on the international level.

\section{EU classification of carcinogens:}

\section{3 carcinogen categories:}

Carcinogen category 1 -shown to cause cancer in humans

Carcinogen category 2-causes cancer in animal tests, and most probably also in humans

Carcinogen category 3-possibly carcinogenic, but evidence supporting carcinogenicity is inadequate for the classification to category 2. 


\section{CARCINOGENS:}

IARC class 1-The substance is carcinogenic to humans.

IARC class $2 \mathrm{~A}$-The substance s probably carcinogenic to humans.

IARC class $2 \mathrm{~B}$-The substance is possibly carcinogenic to humans.

IARC class 3-The substance is not classifiable to as to its carcinogenicity to humans.

IARC class 4-The substance is probably not carcinogenic to humans.

Potency of the carcinogen

- TD25 value

- used for example in the setting of EU OELs for genotoxic carcinogens

- TD25/1000 is considered as an acceptable risk level for genotoxic carcinogens, although also socioeconomic and technical constrains have to be taken into account in the setting of OELs

Non-genotoxic carcinogens - setting of OELs

- No-observed-adverse-effect level (NOAEL) or Lowest-observed-adverse-effect-level (LOAEL)

- uncertainty factor

Different types of carcinogens - OELs and cancer risk

- Genotoxic carcinogens

- no threshold, no zero risk

- even if exposure levels in the workplace are below OEL, we cannot say that there isn't any risk, because according to the current view even small amounts of genotoxic carcinogens may increase our "mutation burden" and our susceptibility to cancer

- therefore, minimization of exposure as far as possible is essential

Different types of carcinogens - OELs and cancer risk

- Non-genotoxic carcinogens

- usually considered to possess a threshold

- for example carcinogens which cause cancer via a mechanism involving chronic injury and regeneration $\Rightarrow>$ if the OEL is set at the level in which no chronic tissue injury is seen, the cancer risk can be regarded to be negligible .

Examples

- Strong inorganic mists of sulphuric acid (IARC class 1)

- Excess risk of laryngeal cancer in workers exposed to sulphuric acid in steel industry.

- Mechanism of action is chronic irritation -caused tissue injury to respiratory tract resulting in reactive stimulation of growth and promotion of cancer.

- air levels of $3-4 \mathrm{mg} / \mathrm{m} 3$ are irritating to the respiratory tract, at lower exposure levels $(0.5-2 \mathrm{mg} / \mathrm{m} 3)$ only mild effects like sensation of acidic taste in the mouth have been reported

Sulphuric acid,

- exposure levels which do not cause irritation can be regarded to protect from carcinogenicity

- e.g. in Finland OEL for sulphuric acid is $0.2 \mathrm{mg} / \mathrm{m} 3 / 8 \mathrm{~h}$ and $1 \mathrm{mg} / \mathrm{m} 3 / 15 \mathrm{~min}$

-Formaldehyde

- a weak genotoxic agent, but its local carcinogenic potential is considered to be mediated mainly via the mechanism involving chronic injury and regeneration

- Anticancer agents like cyclophosphamide

- are known to cause secondary cancers in cancer patients and tumours in experimental animal 
At present, the FDA accepts the three-test package as required by the Ministry of Health and Welfare (MHW) in Japan. In European Community (EC) mutagenicity data are required for the pharmaceuticals before the commencement of clinical trials and the marketing authorization. However, it has further suggested that additional test might be required in specific circumstances. In Japan, the Mini s t r y of $\mathrm{He}$ a l th a n d W e $1 \mathrm{f} \mathrm{a} \mathrm{r} \mathrm{e} \mathrm{(} \mathrm{M} \mathrm{H} \mathrm{W} \mathrm{)} \mathrm{a} \mathrm{d} \mathrm{o} \mathrm{p} \mathrm{t} \mathrm{e} \mathrm{d} \mathrm{mutagenicity} \mathrm{tests} \mathrm{in} 1984$ as one of the several toxicity studies required for the approval to manufacture or import of new drugs.

Definition of 'impurity'

Any Component of the New Drug Substance which is not the Chemical Entity defined as the New Drug Substance. Any Component of the Drug Product which is not the Chemical Entity defined as the drug Substance or an Excipient in the Drug Product."

\section{Classification of Impurities}

- Organic Impurities (Process- and Drug Related)

- Inorganic Impurities

- Residual Solvents

- Polymorphic Forms

- Enantiomeric Impurities

Organic Impurities (Process- and Drug Related)

Organic Impurities can arise during the manufacturing process or storage of the API. They can be Identified or unidentified, volatile or non-volatile.

e.g.:

Starting materials

By-Products

Intermediates

Degradation Products

Reagents, ligands and catalyst

Inorganic Impurities

Inorganic impurities can result from the manufacturing process, they are normally known and identified and include e.g.:

Reagents, Ligands, Catalyst

Heavy Metals or other residual metals

Inorganic Salts

Other material e.g. Filter aids, charcoal

Residual Solvents

Classification, limits and Specification

Class I - Benzene, Carbontetrachloride, 1, 2, dichloroethane etc.

Known human carcinogens, and environmental hazards Solvents to be avoided

Class II - Acetonitrile, Chloroform, methanol, toluene etc.

Non- Genotoxic animal carcinogens, Solvents suspected of significant but reversible toxicities Solvents to be limited.

Class III- Acetone, Butanol, Ethyl acetate, Isopropanol etc.

Solvents with Low toxic potential to humans, no health - based exposure limit needed

Solvents with Low toxic Potential.

Genotoxic Impurities

Definition: Chemical Substances capable of causing direct to indirect damage to DNA or Chromosome and lead to change in the expression of "Gene" thereby leading to Mutated Gene.

Formation of defective Protein

Create Disorder in Metabolic processes

Affect the DNA repair Mechanism

Examples: Alkylating agents, nitoso groups, lead, arsenic etc 
Importance of Genotoxicity \& S2A guidelines for genotoxicity testing for pharmaceuticals "Milestones" in pharmaceutical Regulatory Genetic Toxicity testing:

\begin{tabular}{|l|l|}
\hline $\begin{array}{c}1973,1975 \\
\text { Ames et al }\end{array}$ & "Carcinogens are mutagens" \\
\hline 1980 & CPMP guidelines \\
\hline 1981 & DHSS guidelines \\
\hline $1983-6$ & OECD guidelines \\
\hline 1984 & EEC guidelines \\
\hline 1985 & $2^{\text {nd }}$ IPC Collaborative trial \\
\hline 1989 & Revised DoH guidelines \\
\hline 1995 & ICH Topic S2A \\
\hline 1997 & $\begin{array}{l}\text { ICH Topic S2B } \\
\text { ICH Topic M3 }\end{array}$ \\
\hline 2007 & ICH S2 Revision: Step 2 expected October \\
\hline
\end{tabular}

\section{S2A: Guidance on Specific Aspects of Regulatory Genotoxicity Tests for Pharmaceuticals}

These ICH guidances have been developed by the appropriate ICH Expert Working Group and have been subject to consultation by the regulatory parties, in accordance with the ICH Process. The ICH Steering Committee has endorsed the final draft and recommended its adoption by the regulatory bodies of the European Union, Japan and USA.

Guidelines for the testing of pharmaceuticals for genetic toxicity have been established in the European Community (EEC, 1987) and Japan (Japanese Ministry of Health and Welfare, 1989).FDA's Centres for Drug Evaluation and Research and Biologics Evaluation and Research (CDER and CBER) currently consider the guidance on genetic toxicity testing provided b FDA's Centre for Food Safety and Applied Nutrition (58 FR16536, March 29, 1993) to be applicable to pharmaceuticals.

\section{REGULATORY REQUIREMENTS FOR PHARMACEUTICALS}

\section{ICH Topics:}

- $\quad$ S2A:Guidance on Specific aspects of Regulatory Tests for Pharmaceuticals(Adopted by CPMP September 1995; published in Federal Register April 1996)

- $\quad$ S2B:Genotoxicity: a Standard Battery for Testing of Pharmaceuticals(Adopted by CPMP September 1997; published in Federal Register November 1997)

- M3:Timing of Pre-Clinical Studies in Relation to Clinical Trials(Adopted by CPMP September 1996; published in Federal Register November 1997)

\section{$>\quad$ ICH Topic S2B}

- The Standard Test Battery for Genotoxicity

i) A test for gene mutation in bacteria

ii) An invitro test for cytogenetic evaluation of chromosomal damage with mammalian cells OR An in vitro mouse lymphoma TK assay.

iii) An in vivo test for chromosomal damage using rodent haematopoietic cells

\section{$>\quad$ ICH Topic M3}

- Genotoxicity studies

Prior to first human exposure, in vitro tests for the evaluation of mutations and chromosome damage are really needed. If an equivocal or positive finding occurs, additional testing should be performed.

The standard battery of tests for genotoxicity (Topic S2B) should be completed prior to initiation of phase II studies.

\section{SPECIFIC GUIDANCE AND RECOMMENDATIONS}

\section{A. Specific guidance for in vitro tests}

1. The base set of strains used in bacterial mutation assays.

Current guidelines for the detection of bacterial mutagens employ several strains to 
Importance of Genotoxicity \& S2A guidelines for genotoxicity testing for pharmaceuticals

Detect base substitution and frame shift point mutations. The Salmonella and TA100) will detect such changes at G-C (guanine-cytosine) sites within target histidine genes. It is clear from the literature that some mutagenic carcinogens also modify A-T (adenine-thymine) base pairs. Therefore, the standard set of strains used in bacterial mutation assays should include strains that will detect point mutations at A-T sites, such as S. typhimurium TA102, which detects such mutations within multiple copies of hisG genes, or Escherichia coli WP2 uvrA, which detects these mutations in the trpE gene, or the same strain possessing the plasmid (pKM101), which carries mucAB genes that enhance error prone repair. In conclusion, the following base set of bacterial strains should be used for routine testing: The strains cited below are all S. typhimurium isolates, unless specified otherwise.

1. TA98; 2. TA100; 3. TA1535; 4. TA1537 or TA97 or TA97a2; 5. TA102 or E. coli WP2 uvrA or E. coli WP2 uvrA (pKM101).

In order to detect cross-linking agents it may be preferable to select $\mathrm{S}$.

Typhimurium TA 102 or to add a repair proficient E. coli strain, such as WP2 pKM101. It is noted that such compounds are detected in assays that measure chromosome damage.

\section{Ames test}

- Uses strains of Salmonella typhimurium and Escherichia coli

- Strains have defined mutations making them require specific amino acids

- S. typhimurium -histidine,E. coli -tryptophan

- Back mutation allows growth in medium without histidine or tryptophan
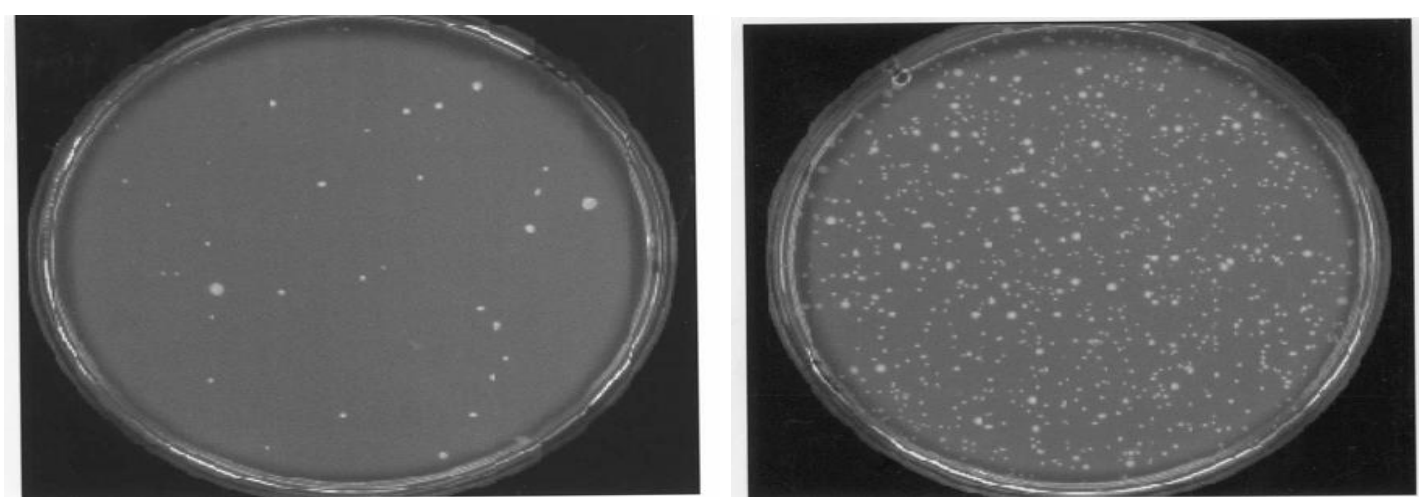

Bacteria ( $2 / 5$ strains) removed from, freezer and incubated shaking $(37 \mathrm{oC})$ overnight

Serial dilution of test compound

\&

Metabolic activation system (Rat liver S9 fraction and co-factors)

Test compound, S9, top agar and bacteria mixed \& poured onto agar plate (trace histidine/tryptophan). Three plates per concentration

Plates incubated at $37 \mathrm{oC}$ for 3 days

Mutant (revertant) colonies can grow without histidine .Number of colonies per plate counted.

2. Definition of the top concentration for in vitro tests

a. High concentration for non-toxic compounds

For freely soluble, non-toxic compounds, the desired upper treatment levels are 5 milligrams $(\mathrm{mg}) / \mathrm{plate}$ for bacteria and $5 \mathrm{mg} / \mathrm{millilitre}(\mathrm{mL})$ or 10 mill molar $(\mathrm{mM})$ (whichever is the lower) for mammalian cells.

b. Desired level of cytotoxicity 
Some genotoxic carcinogens are not detectable in in vitro genotoxicity assays unless the concentrations tested induce some degree of cytotoxicity. It is also apparent that excessive toxicity often does not allow a proper evaluation of the relevant genetic endpoint. Indeed, at very low survival levels in mammalian cells, mechanisms other than direct genotoxicity per se can lead to "positive" results that are related to cytotoxicity and not genotoxicity (e.g., events associated with apoptosis, endonuclease release from lysosomes, etc.). Such events are likely to occur once a certain concentration threshold is reached for a toxic compound. To balance these conflicting considerations, the following levels of cytotoxicity are currently considered acceptable for in vitro bacterial and mammalian cell tests (concentrations should not exceed the levels specified

In:

i. In the bacterial reverse mutation test, the highest concentration of test compound is desired to show evidence of significant toxicity. Toxicity may be detected by a reduction in the number of revertants, a clearing or diminution of the background

lawn.

\section{Strain}

TA $1535 \&$ TA 100

TA 1537 \& TA98

E.coli uvrA pKM101

\author{
Reversion event \\ Base -pair substitution \\ Frame-shift \\ Base-pair substitution \\ Excision-repair proficient \\ (detects cross-linking agents)
}

ii. The desired level of toxicity for in vitro cytogenetic tests using cell lines should be greater than 50 percent reduction in cell number or culture confluency. For lymphocyte cultures, an inhibition of mitotic index by greater than 50 percent is considered sufficient.

iii. In mammalian cell mutation tests, ideally the highest concentration should produce at least 80 percent toxicity (no more than 20 percent survival). Toxicity can be measured either by assessment of cloning efficiency (e.g., immediately after treatment), or by calculation of relative total growth, i.e., the product of relative suspension growth during the expression period and relative plating efficiency at the time of mutant selection. Caution is due with positive results obtained at levels of survival lower than 10 percent.

c. Testing of poorly soluble compounds.

There is some evidence that dose-related genotoxic activity can be detected when testing certain compounds in the insoluble range in both bacterial a mammalian cell genotoxicity tests. This is generally associated with doserelated toxicity. It is possible that solubilization of a precipitate is enhanced by serum in the culture medium or in the presence of S9-mix constituents. It is also probable that cell membrane lipid can facilitate absorption of lipophilic compounds into cells. In addition, some types of mammalian cells have endocytic activity (e.g., Chinese hamster V79, $\mathrm{CHO}$ and CHL cells) and can ingest solid particles that may subsequently disperse into the cytoplasm. An insoluble compound may also contain soluble genotoxic impurities. It should also be noted that a number of insoluble pharmaceuticals are administered to humans as suspensions or as particulate materials. On the other hand, heavy precipitates can interfere with scoring the desired parameter and render control of exposure very difficult (e.g., where centrifugation step(s) is (are) included in a protocol to remove cells from exposure media), or render the test compound unavailable to enter cells and interact with DNA.The following strategy is recommended for testing relatively insoluble compounds. The recommendation below refers to the test article in the culture medium.

If no cytotoxicity is observed, then the lowest precipitating concentration should be used as the top concentration but not exceeding $5 \mathrm{mg}$ per plate for bacterial tests and $5 \mathrm{mg} / \mathrm{mL}$ or $10 \mathrm{mM}$ for mammalian cell tests. If dose-related cytotoxicity or mutagenicity is noted, irrespective of solubility, then the top concentration should be based on toxicity as described above. This may require the testing of more than one precipitating concentration (not to exceed the above stated levels). It is recognized that the desired levels of cytotoxicity may not be achievable if the extent of precipitation interferes with the scoring of the test. In all cases, precipitation should be evaluated at the beginning and at the end of the treatment period using the naked eye. 


\section{B. Specific guidance for in vivo tests}

\section{$>$ Rodent bone-marrow micronucleus test}

Rats/mice dosed with compound, three doses, seven animals / group. Animals sacrificed 24 or 48 hours later

Micronuclei may be formed by loss of whole chromosome during division or by chromosome breakage. The erythrocyte's nucleus is extruded leaving any micronuclei behind

Femurs removed and bone marrow aspirated

Bone marrow cells spread onto slides. Slides fixed and stained (acridine orange)

2000 cells analysed per animal, number of micro nucleated immature erythrocytes scored.

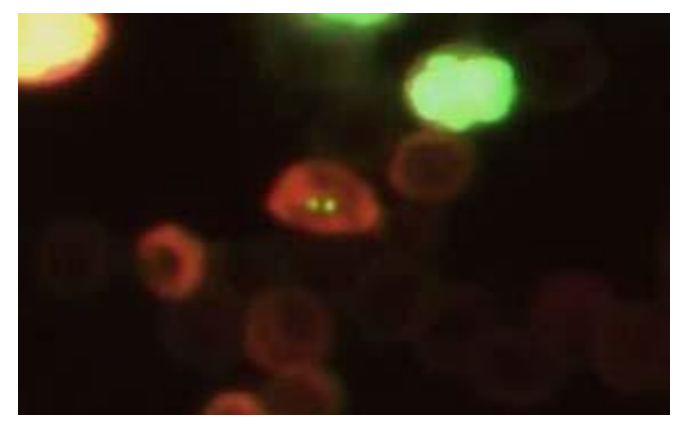

1. Acceptable bone marrow tests for the detection of clastogens in vivo Tests measuring chromosomal aberrations in nucleated bone marrow cells in rodents can detect a wide spectrum of changes in chromosomal integrity. These changes almost all result from breakage of one or more chromatids as the initial event. Breakage of chromatids or chromosomes can result in micronucleus formation if an acentric fragment is produced; therefore, assays detecting either chromosomal aberrations or micronuclei are acceptable for detecting clastogens.Micronuclei can also result from lagging of one or more whole chromosome(s) at anaphase and thus micronucleus tests have the potential to detect some aneuploidy inducers. In conclusion, either the analysis of chromosomal aberrations in bone marrow cells or the measurement of micro nucleated polychromatic erythrocytes in bone marrow cells in vivo is acceptable for the detection of clastogens. The measurement of micro nucleated immature (e.g., polychromatic) erythrocytes in peripheral blood is an acceptable alternative in the mouse, or in any other species in which the inability of the spleen to remove micro nucleated erythrocytes has been demonstrated, or which has shown an adequate sensitivity to detect clastogens/aneuploidy inducers in peripheral blood.

2. Use of male/female rodents in bone marrow micronucleus tests.

Extensive studies of the activity of known clastogens in the mouse bone marrow micronucleus test have shown that, in general, male mice are more sensitive than female mice for micronucleus induction. Quantitative differences in micronucleus induction have been identified between the sexes, but no qualitative differences have been described. Where marked quantitative differences exist, there is invariably a difference in toxicity between the sexes. If there is a clear qualitative difference in metabolites between male and female rodents, then both sexes should

be used. Similar principles can be applied for other established in vivo tests. Both rats and mice are deemed acceptable for use in the bone marrow micronucleus test. 
male and female rodents, males alone are sufficient for use in bone marrow micronucleus tests. If genderspecific drugs are to be tested, animals of the corresponding sex should normally be used.

\section{$>$ Rodent bone-marrow micronucleus test OECD Guideline 474}

Highest dose tested: $2000 \mathrm{mg} / \mathrm{kg}$ (free base) MTD

Amount of compound required Up to $20 \mathrm{~g}$ depending on MTD

Detects: chromosome breakage chromosome loss (aneuploidy)

Detection of rodent carcinogens by standard in vitro tests

\begin{tabular}{|l|l|l|l|}
\hline & Ames & MLA & Ames + MLA \\
\hline Sensitivity & $318 / 541$ & $179 / 245$ & $389 / 436$ \\
& $59 \%$ & $73 \%$ & $89 \%$ \\
\hline specificity & $130 / 176$ & $41 / 105$ & $34 / 105$ \\
& $74 \%$ & $39 \%$ & $32 \%$ \\
\hline
\end{tabular}

Sensitivity=proportion of carcinogens giving positive results Specificity=proportion on non-carcinogens giving negative results.

\section{Guidance on the evaluation of test results}

Comparative trials have shown conclusively that each in vitro test system generates both false negative and false positive results in relation to predicting rodent carcinogenicity. Genotoxicity test batteries (of in vitro and in vivo tests) detect carcinogens that are thought to act primarily via a mechanism involving direct genetic damage, such as the majority of known human carcinogens. Therefore, these batteries may not detect

nongenotoxic carcinogens. Experimental conditions, such as the limited capability of the in vitro metabolic activation systems, can also lead to false negative results in in vitro tests. The test battery approach is designed to reduce the risk of false negative results for compounds with genotoxic potential, while a positive result in any assay for genotoxicity does not necessarily mean that the test compound poses a genotoxic/carcinogenic hazard to humans.

\section{Guidance On The Evaluation Of In Vitro Test Results a. In vitro positive results}

The scientific literature gives a number of conditions that may lead to a

positive in vitro result of questionable relevance. Therefore, any in vitro positive test result should be evaluated for its biological relevance taking

into account the following considerations (this list is not exhaustive, but is given as an aid to decision-making):

i. Is the increase in response over the negative or solvent

control background regarded as a meaningful genotoxic effect for

the cells?

ii. Is the response concentration-related?

iii. For weak/equivocal responses, is the effect reproducible?

iv. Is the positive result a consequence of an in vitro specific

metabolic activation pathway/in vitro specific active metabolite? 12

v. Can the effect be attributed to extreme culture conditions that do not occur in in vivo situations, e.g., extremes of $\mathrm{pH}$; osmolality; heavy precipitates, especially in cell suspensions?

vi. For mammalian cells, is the effect only seen at extremely low survival levels?

vii. Is the positive result attributable to a contaminant (this may be the case if the compound shows no structural alerts or is weakly mutagenic or mutagenic only at very high concentrations)?

viii. Do the results obtained for a given genotoxic endpoint

conform to that for other compounds of the same chemical class?

\section{b. In vitro negative results}

For in vitro negative results, special attention should be paid to the following considerations (the examples given are not exhaustive, but are given as an aid to decision-making): Does the structure or known metabolism of the compound indicate that standard techniques for in vitro metabolic activation (e.g., rodent liver S9) may be inadequate? Does the structure or known reactivity of the compound indicate that the use of other test methods/systems may be appropriate? 


\section{Guidance On The Evaluation Of In Vivo Test Results}

In vivo tests, by their nature, have the advantage of taking into account absorption, distribution, and excretion, which are not factors in in vitro tests, but are relevant to human use. In addition, metabolism is likely to be more relevant in vivo compared to the systems normally used in vitro. There are a few validated in vivo models accepted for assessment of genotoxicity. These include the bone marrow or peripheral blood cytogenetic assays. If a compound has been tested in vitro with negative results, it is usually sufficient to carry out a single in vivo cytogenetics assay. For a compound that induces a biologically relevant positive result in one or more in vitro tests, a further in vivo test in addition to the in vivo cytogenetic assay, using a tissue other than the bone marrow/peripheral blood, can provide further useful information. The target cells exposed in vivo and possibly the genetic end point measured in vitro guide the choice of this additional in vivo test. However, there is no validated, widely used in vivo system that measures gene mutation. In vivo gene mutation assays using endogenous genes or transgenes in several tissues of the rat and mouse are at various stages of development. Until such tests for mutation become accepted, results from other in vivo tests for genotoxicity in tissues other than the bone marrow can provide valuable additional data but the assay of choice should be scientifically justified. If in vivo and in vitro test results do not agree, then the differences should be considered/explained on a case-by-case basis.

In conclusion, the assessment of the genotoxic potential of a compound should take into account the totality of the findings and acknowledge the intrinsic values and limitations of both in vitro and in vivo tests.

a. Principles for demonstration of target tissue exposure for negative

in vivo test results. In vivo tests have an important role in genotoxicity test strategies. The significance of in vivo results in genotoxicity test strategies is directly related to the demonstration of adequate exposure of the target tissue to the test compound. This is especially true for negative in vivo test results and when in vitro test(s) have shown convincing evidence of genotoxicity. Although a dose sufficient to elicit a biological response (e.g., toxicity) in the tissue in question is preferable, such a dose could prove to be unattainable since dose-limiting toxicity can occur in a tissue other than the target tissue of interest. In such cases, toxicokinetic data can be used to provide evidence of bioavailability. If adequate exposure cannot be achieved, e.g., with compounds showing very poor target tissue availability, extensive protein binding, etc., conventional in vivo genotoxicity tests may have little value. The following recommendations apply to bone marrow cytogenetic assays; as examples, if other target tissues are used, similar principles should be applied.

For compounds showing positive results in any of the in vitro tests employed demonstration of in vivo exposure should be made by any of the following measurements:

i. By obtaining a significant change in the proportion of immature erythrocytes among total erythrocytes in the bone marrow, at the doses and sampling times used in the micronucleus test or by measuring a significant reduction in mitotic index for the

chromosomal aberration assay.

ii. Evidence of bioavailability of drug-related material either by measuring blood or plasma levels.

iii. by direct measurement of drug-related material in bone marrow.

iv. By auto radiographic assessment of tissue exposure.

For methods (ii) to (iv), assessments should be made preferentially at the top dose or other relevant doses using the same species/strain and dosing route used in the bone marrow assay. If in vitro tests do not show genotoxic potential, in vivo (systemic) exposure should be demonstrated and can be achieved by any of the methods above, but can also be inferred from the results of standard absorption, distribution, metabolism, and excretion studies in rodents.

b. Detection of germ cell mutagens.

With respect to the detection of germ cell mutagens, results of comparative studies have shown that, in a qualitative sense, most germ cell mutagens are likely to be detected as such in somatic cell tests and negative results of in vivo somatic cell genotoxicity tests generally indicate the absence of germ cell effects. 
FLOWCHART FOR GENOTOXICITY TESTING:

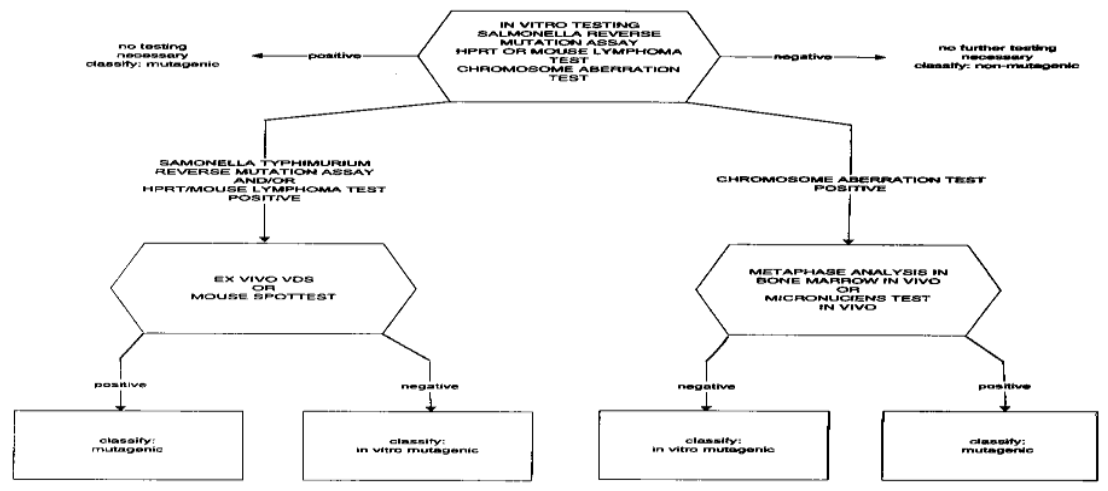

\section{$>$ IMPURITIES -CURRENT LEGISLATION AND PROPOSALS}

- ICH Q3A (R) -Impurities in New Drug Substances (2002)

- $\quad$ ICH Q3B (R) -Impurities in New Drug Products (2006)

- CHMP Guideline on the Limits of Genotoxic Impurities (2006)

\section{Further guidance required:}

- ICH Q3A/B do not provide sufficient confidence for genotoxic impurities

- CHMP does not consider compounds in development

- PhRMA rationale

\section{$>$ CHMP Guideline on the Limits of Genotoxic Impurities}

Assumes no thresholds for DNA reactive agents.

Separates potential genotoxic impurities (PGI) into those with and without sufficient experimental evidence for threshold-related mechanisms e.g.

spindle disrupters

topoisomerase inhibitors

inhibitors of DNA synthesis

etc

Impossible to define "safe" exposure to non-threshold genotoxins

Pragmatic "Threshold of Toxicological Concern"

\section{Threshold of Toxicological Concern (TTC) -origins}

- 1958 Delaney Clause(amendment to 1954 US Federal Food, Drug and Cosmetic Act) No food additive can be deemed safe, or given approval, if found to cause cancer in animals or man

- Justification -experts unable to set absolutely safe levels for any carcinogen

- $\quad 1980$ 's -improvements in analytical technologies showed quantifiable traces of numerous substances in food 
Importance of Genotoxicity \& S2A guidelines for genotoxicity testing for pharmaceuticals

- $\quad 1979$-US Court case involving Monsanto over leaching of a polymer from a drinks container allowed FDA to accept a negligible risk level of contamination

- $\quad$ Original work by Rulis followed by Gold, then Munro

Low probability that $1 \mathrm{ppb}$ in diet will present a lifetime cancer risk $\quad>1$ in 106

- $\quad$ FDA "Threshold of Regulation" for trace substances from food contact materials

$0.5 \mathrm{ppb}$ in $3 \mathrm{~kg}$ food per day $\Rightarrow 1.5 \mu \mathrm{g} / \mathrm{day}$

- $\quad$ Carcinogenic contaminants justified without change to Delaney Clause

\section{CHMP Guideline -TTC}

- Linear extrapolation of animal data for 730 carcinogens

- Daily exposure to $\leq 1.5 \mu \mathrm{g} /$ dayfor most carcinogens

should not exceed a lifetime cancer risk of 1 in 106

- $\quad$ High potency carcinogens include:

Aflatoxins

$\mathrm{N}$-nitroso compounds

Azoxy compounds

10-fold lower TTC

- $\quad$ Lifetime risk of 1 in106 is conservative, since drugs have benefit

Therefore, TTC limit based on 1 in 105I.e. $\leq 1.5 \mu \mathrm{g} /$ day

- $\quad$ Higher levels if justifiable e.g.

Acute drug treatment

Life-threatening disease

Lack of alternatives

- $\quad$ Proposed level of acceptable risk consistent with other regulations

\begin{tabular}{|l|l|}
\hline WHO drinking water standards & 1 in $10^{5}$ \\
\hline USEPA & 1 in $10^{4}$ to 1 in $10^{6}$ \\
\hline FAO/WHO flavouring substances & 1 in $10^{6}$ \\
\hline Q3C limit set for benzene,20microgram per day & 1 in $10^{5}$ \\
\hline
\end{tabular}

\section{REFERENCES:}

[1]. 1.'Guidelines for industry-specific aspects for regulatory genotoxicity tests for pharmaceuticals', April 1996

[2]. 2. Mike O'Donovan-safety testing of genotoxic impurities,' what a chemist needs to know about safety assessment', 7th June, 2007.

\section{AUHTOR:}

Ms. SHAILY.U.SHAH

Under the guidance of

Mr.ANIL. GHOGARE Sir. 